\title{
METHOD
}

\section{Caterpillars Count! A Citizen Science Project for Monitoring Foliage Arthropod Abundance and Phenology}

\author{
Allen Hurlbert*, Tracie Hayes*, Tara McKinnon* and Christine Goforth ${ }^{\dagger}$
}

Caterpillars Count! is a citizen science project that allows participants to collect data on the seasonal timing, or phenology, of foliage arthropods that are important food resources for forest birds. This project has the potential to address questions about the impacts of climate change on birds over biogeographic scales. Here, we provide a description of the project's two survey protocols, evaluate the impact of survey methodology on results, compare findings made by citizen scientist participants versus trained scientists, and identify the minimum levels of sampling frequency and intensity needed to accurately capture phenological dynamics. We find that beat sheet surveys and visual surveys yield similar relative and absolute density estimates of different arthropod groups, with beat sheet surveys recording a higher frequency of beetles and visual surveys recording a higher frequency of flies. Citizen scientists generated density estimates within $6 \%$ of estimates obtained by trained scientists regardless of survey method. However, patterns of phenology were more consistent between citizen scientists and trained scientists when using beat sheet surveys than visual surveys. By subsampling our survey data, we found that conducting 30 foliage surveys on a weekly basis led to $95 \%$ of peak caterpillar date estimates to fall within one week of the "true" peak. We demonstrate the utility of Caterpillars Count! for generating a valuable dataset for ecological research, and call for future studies to evaluate how training and resource materials impact data quality and participant learning gains.

Keywords: arthropods; caterpillars; phenology; survey methodology; data validation

One of the observed impacts of climate change over recent decades has been a shift in the seasonal timing, or phenology, of organisms and their life cycles. For example, first flowering dates in Concord, Massachusetts have advanced by two to three weeks since Thoreau's records from the 1850s (Ellwood et al. 2013; Primack 2014). Butterflies have similarly advanced first flight dates over recent decades (Altermatt 2012; Forister and Shapiro 2003), and many bird species have advanced the timing of migration (Hurlbert and Liang 2012; Mayor et al. 2017). Such observed phenological shifts indicate that these species are able to respond to changes in their physical environment, yet the magnitude of these shifts is highly variable among species and across trophic levels (Both et al. 2009; Parmesan 2007; Parmesan and Yohe 2003). Phenological mismatch occurs when organisms fail to adjust their seasonal timing to the same degree as the organisms on which they depend, and has been documented between plants and their pollinators (Forrest 2015), insects and their host plants (Singer and Parmesan 2010), and birds

\footnotetext{
* University of North Carolina, US

+ North Carolina Museum of Natural Sciences, US

Corresponding author: Allen Hurlbert

(hurlbert@bio.unc.edu)
}

and the arthropod food sources on which they rely for successfully raising offspring (Visser et al. 2006, 2012). Understanding phenological mismatch in migratory birds is a particularly challenging problem because these birds often traverse thousands of kilometers, and climate change is geographically variable over these regions. For example, observed phenological shifts in the northeastern US may be poorly correlated with shifts in the southeast, which would have important impacts on migratory birds (Fontaine et al. 2015; Wood and Kellermann 2015).

Citizen science programs are one of the most effective ways to monitor biological phenomena like phenology over broad geographic extents as demonstrated by the recent efforts of the National Phenology Network (Schwartz et al. 2012), Project Budburst (Johnson 2016), and eBird (Sullivan et al. 2014). Individual scientists or research groups are simply unable to collect data efficiently at the relevant spatial and temporal scales for addressing these broad biogeographical questions. Here we introduce a new citizen science project, Caterpillars Count! (https://caterpillarscount.unc.edu), which aims to document geographic and annual variation in the phenology and abundance of arthropods that foliage gleaning birds rely on during the breeding season. The name of the project highlights the fact that Lepidoptera larvae in particular represent an important and often primary food source 
(Holmes et al. 1979; Holmes and Schultz 1988; Jones et al. 2003; Sillett et al. 2000) known to influence avian density (Graber and Graber 1983), reproductive success (Rodenhouse and Holmes 1992; Visser et al. 2006), clutch size (Perrins 1991) and number of broods raised (Nagy and Holmes 2005a, 2005b). The enlistment of citizen scientists would potentially allow for an examination of phenological mismatch between birds and their food resources at an unprecedented scale.

In this paper we describe the survey protocols used to monitor foliage arthropods as part of the Caterpillars Count! project and address three research questions. Specifically, we 1) evaluate the impact of survey methodology on results, 2) compare findings made by citizen scientist participants versus trained scientists to assess the reliability of citizen science data collection and to make recommendations for citizen science coordinators, and 3) identify the minimum levels of sampling frequency and intensity required to accurately capture phenological dynamics. We hope that Caterpillars Count! will yield robust data on arthropod phenology over broad spatial scales, which ultimately can be leveraged with other existing datasets to provide new insights into potential mismatches between vegetation, arthropods, and birds.

\section{Caterpillars Count! Protocol}

Because arthropods may be patchily distributed across an area, accurate estimates of density require conducting many surveys per survey date. Permanent survey branches are arrayed across the study site in groups ("circles") of five, with a central survey branch identified opportunistically (e.g., a branch with additional suitable vegetation nearby) followed ideally by the first suitable branch $5 \mathrm{~m}$ away in each of the four cardinal directions (Figure 1, inset).
To be suitable, a branch must have at least 50 leaves (or leaflets for compound leaves) each greater than $5 \mathrm{~cm}$ in length. The opportunistic selection of the center point for each circle of surveys allows flexibility in survey layout, because in many cases suitable branches may be limited in availability and found only in certain areas within the site. However, by choosing the surrounding branches more systematically we expect the plant species selected to be representative of woody vegetation at the site. Survey circles should be spaced with centers at least $15 \mathrm{~m}$ apart to avoid overlap between circles. However, for some sites that aim to characterize arthropods over a larger area, circles may be up to several hundred meters apart. Other considerations at the discretion of the site coordinator are whether survey branches should be located off trail to minimize unwanted disturbance of survey branches, or along trails to minimize off-trail impacts by volunteers.

To participate in the project, a site must establish at least two survey circles (10 total surveys), but some sites have as many as 12 circles (60 total surveys). We allow flexibility in the total number of surveys established at a site because while a greater number of surveys yields better estimates of arthropod density, not all sites have the human resources to conduct a large number of surveys. One aim of this manuscript is to evaluate how many surveys are required to provide meaningful characterizations of arthropod phenology. Sites that participate at a lower level may be excluded from certain analyses.

\section{Visual foliage survey}

Visual foliage surveys conducted at ground level have been used for decades to characterize foliage arthropod availability to birds throughout the forest canopy (Holmes and Schultz 1988). For one survey, an observer

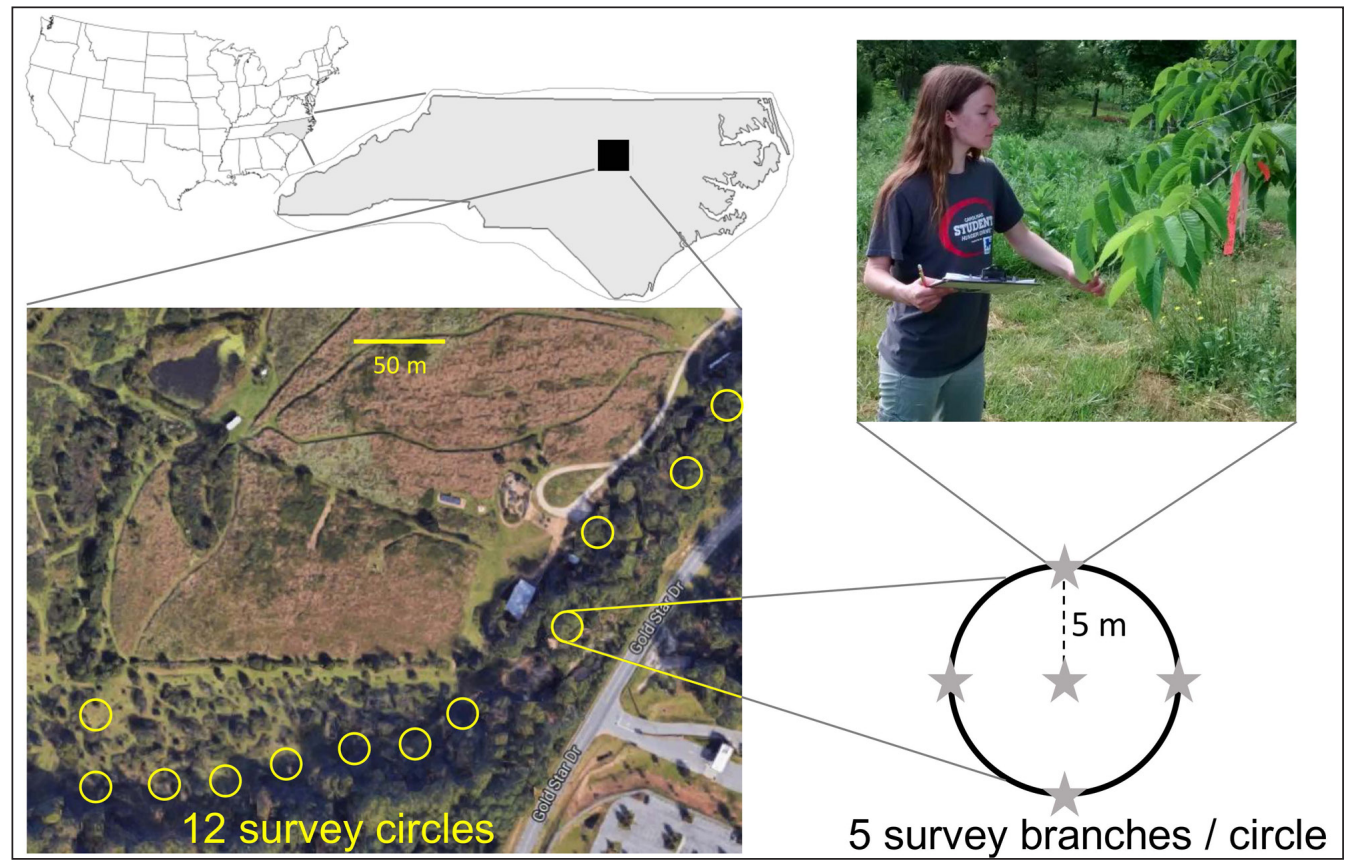

Figure 1: Location of Prairie Ridge Ecostation within North Carolina, and the layout of survey circles at that site. Each survey circle consists of five foliage arthropod surveys. Photo credits: Google Earth; Allen Hurlbert. 
examines both the upper- and undersides of 50 leaves and associated petioles and twigs on a branch of woody vegetation typically $1-2 \mathrm{~m}$ above the ground. All arthropods observed greater than $2 \mathrm{~mm}$ in length are identified, generally to order (but in some cases suborder or family; Table 1), and their body length (not including legs or antennae) is recorded to the nearest millimeter. Arthropods smaller than $2 \mathrm{~mm}$ are ignored both because of their lesser importance as food items as well as the increased difficulty and therefore time required for identification. A single visual foliage survey takes 2-6 minutes depending upon the density of arthropods, experience of the observer, and degree of clustering of leaves on a branch.

\section{Beat sheet survey}

As an alternative to the visual foliage survey, participants may choose instead to conduct a beat sheet survey in which the survey branch is beaten with a stick ten times in rapid succession over a white $60 \times 60 \mathrm{~cm}$ sheet. As with the visual survey, all arthropods are identified to the relevant order/group (Table 1) and length is recorded to the nearest millimeter. In addition, the participant records the total number of leaves that were positioned above the beat sheet during beating which is expected to vary from branch to branch. A single beat sheet survey typically takes 2-3 minutes depending on the density of arthropods and the experience of the observer.

\section{Methods}

\section{Data collection}

Foliage arthropod surveys were conducted at Prairie Ridge Ecostation $\left(35.8117^{\circ} \mathrm{N}, 78.7139^{\circ} \mathrm{W}\right)$, an outdoor nature center in Raleigh, NC. This site features a narrow strip of deciduous forest, with a diverse tree community including Liquidambar styraciflua, Acer negundo, and Diospyros virginiana, alongside an open prairie. Sixty survey locations were established in 12 groups ("circles") of five surveys each (Figure 1).

In both 2015 and 2016, members of the Hurlbert Lab at the University of North Carolina (hereafter "trained scientists") conducted visual and beat sheet surveys twice per week from mid-May through July at all survey locations (Table 2). With the exception of Hurlbert, trained scientists were undergraduate students with no significant entomological expertise prior to participating. Hurlbert conducted surveys himself but also provided extensive training before and during all foliage survey activities throughout the season, ensuring that team members properly followed survey protocols and were capable of documenting potentially cryptic arthropods and of identifying arthropods to the relevant groups. Visual surveys were conducted first at each survey location followed by a beat sheet survey on an adjacent branch of the same plant species. Surveys were typically conducted between 0830 and 1200 hrs. In 2015, trained scientists additionally conducted beat sheet surveys once per week on Thursday

Table 1: Common arthropod groups found on foliage that citizen scientist participants are expected to be able to identify.

\begin{tabular}{|c|c|c|c|}
\hline Common name & Scientific name & Taxonomic level & Distinguishing features \\
\hline Ants & Formicidae & Family & Narrow waist, no wings; elbowed antennae. \\
\hline Aphids, Psyllids & Sternorrhyncha & $\begin{array}{l}\text { Suborder, Order } \\
\text { Hemiptera }\end{array}$ & Small (just a few mm); aphids are pear-shaped. \\
\hline Bees, Wasps & $\begin{array}{l}\text { Hymenoptera (exclud- } \\
\text { ing Formicidae) }\end{array}$ & Order & $\begin{array}{l}2 \text { pairs of wings with the hindwings smaller than } \\
\text { the frontwings; wasps have narrow waists but } \\
\text { bees do not. }\end{array}$ \\
\hline Beetles & Coleoptera & Order & $\begin{array}{l}\text { A straight line down the back where the two hard } \\
\text { wing casings (elytra) meet. }\end{array}$ \\
\hline Caterpillars & Lepidoptera (larvae) & Order & $\begin{array}{l}\text { Soft, cylindrical body with } 6 \text { legs and up to } 5 \text { pairs } \\
\text { of prolegs. }\end{array}$ \\
\hline Daddy longlegs & Opiliones & Order & $\begin{array}{l}8 \text { very long legs; they appear to have a single } \\
\text { oval-shaped body. }\end{array}$ \\
\hline Flies & Diptera & Order & A single pair of wings. \\
\hline Grasshoppers, Crickets & Orthoptera & Order & Usually with enlarged hind legs for jumping. \\
\hline Leafhoppers, Cicadas & Auchenorrhyncha & $\begin{array}{l}\text { Suborder, Order } \\
\text { Hemiptera }\end{array}$ & $\begin{array}{l}\text { Usually a wide head relative to the body; hoppers } \\
\text { have wings folded tentlike over their back, while } \\
\text { cicadas have large membranous wings. }\end{array}$ \\
\hline Moths, Butterflies & Lepidoptera (adults) & Order & 4 large wings covered by fine scales. \\
\hline Spiders & Araneae & Order & $\begin{array}{l}8 \text { legs, with two distinct body segments: the } \\
\text { cephalothorax and abdomen. }\end{array}$ \\
\hline True Bugs & Heteroptera & $\begin{array}{l}\text { Suborder, Order } \\
\text { Hemiptera }\end{array}$ & $\begin{array}{l}\text { Semi-transparent wings which partially overlap } \\
\text { creating a triangle or X shape on the back; often } \\
\text { has pointy "shoulders". }\end{array}$ \\
\hline
\end{tabular}


afternoons, typically between 1300 and 1400, at a fixed subset of 40 of the 60 total survey locations. This was done to check for an effect of time of day on the observed phenological patterns, but no strong effect on phenological timing was found (Figure 2).

Also in both 2015 and 2016, volunteers (hereafter "citizen scientists") were recruited to conduct foliage arthropod surveys at the fixed subset of 40 survey locations (Table 2). Citizen scientists were recruited through the volunteer program at the North Carolina Museum of Natural Sciences and included both men and women varying in age from 22 to 50 years in age. Volunteers were trained by CLG, who worked with the volunteers the first three times they conducted surveys and focused heavily on arthropod identification skill building. After the third survey, the volunteers conducted the surveys on their own. Thus, while citizen scientists and trained scientists received similar training initially, they differed in the duration of supervision in the field. In 2015, seven different citizen scientists conducted visual foliage surveys, some on Thursdays between 1300 and 1500 and others on Saturdays between 0900 and 1100 hours most weeks. In 2016, four citizen scientists were recruited, and they conducted beat sheet surveys once per week on average, typically between 0800 and 1200 hrs. We were thus able to compare citizen scientist and trained scientist observations based on visual surveys in 2015, and based on beat sheet surveys in 2016. Citizen scientists conducted 166-185 surveys per participant on average over the course of each season, while trained scientists conducted 1111-1662 surveys per participant on average and so had more experience on top of the increased training and supervision (Table 2).

Finally, while the Caterpillars Count!survey methodology focused on foliage 1-2 $\mathrm{m}$ above ground for logistical reasons, we would ideally like to make inferences about arthropod phenology throughout the entire canopy. To validate this comparison between foliage strata, we collected caterpillar frass falling from the canopy in 2015 to compare with observed phenology from the ground level foliage surveys. Although citizen scientists did not collect frass data, it is crucial to understand whether this citizen

Table 2: Number of participants and number of surveys conducted from May through July for each data collection group and year. Survey method abbreviations: V, visual survey; B, beat sheet survey; F, frass monitoring.

\begin{tabular}{lllrrrl} 
Group & Year & $\begin{array}{l}\text { Survey } \\
\text { method }\end{array}$ & $\begin{array}{c}\text { \# of par- } \\
\text { ticipants }\end{array}$ & $\begin{array}{c}\text { \# surveys conducted } \\
\text { per person }\end{array}$ & $\begin{array}{l}\text { Total \# } \\
\text { surveys }\end{array}$ & $\begin{array}{l}\text { Time of } \\
\text { day }\end{array}$ \\
\hline Trained scientists & 2015 & $\mathrm{~V}, \mathrm{~B}, \mathrm{~F}$ & 5 & 1662 & 8310 & am, pm \\
Citizen scientists & 2015 & $\mathrm{~V}$ & 7 & 166 & 1165 & $\mathrm{am}, \mathrm{pm}$ \\
Trained scientists & 2016 & $\mathrm{~V}, \mathrm{~B}$ & 5 & 1111 & 5553 & $\mathrm{am}$ \\
Citizen scientists & 2016 & $\mathrm{~B}$ & 4 & 185 & $739 \mathrm{am}$
\end{tabular}

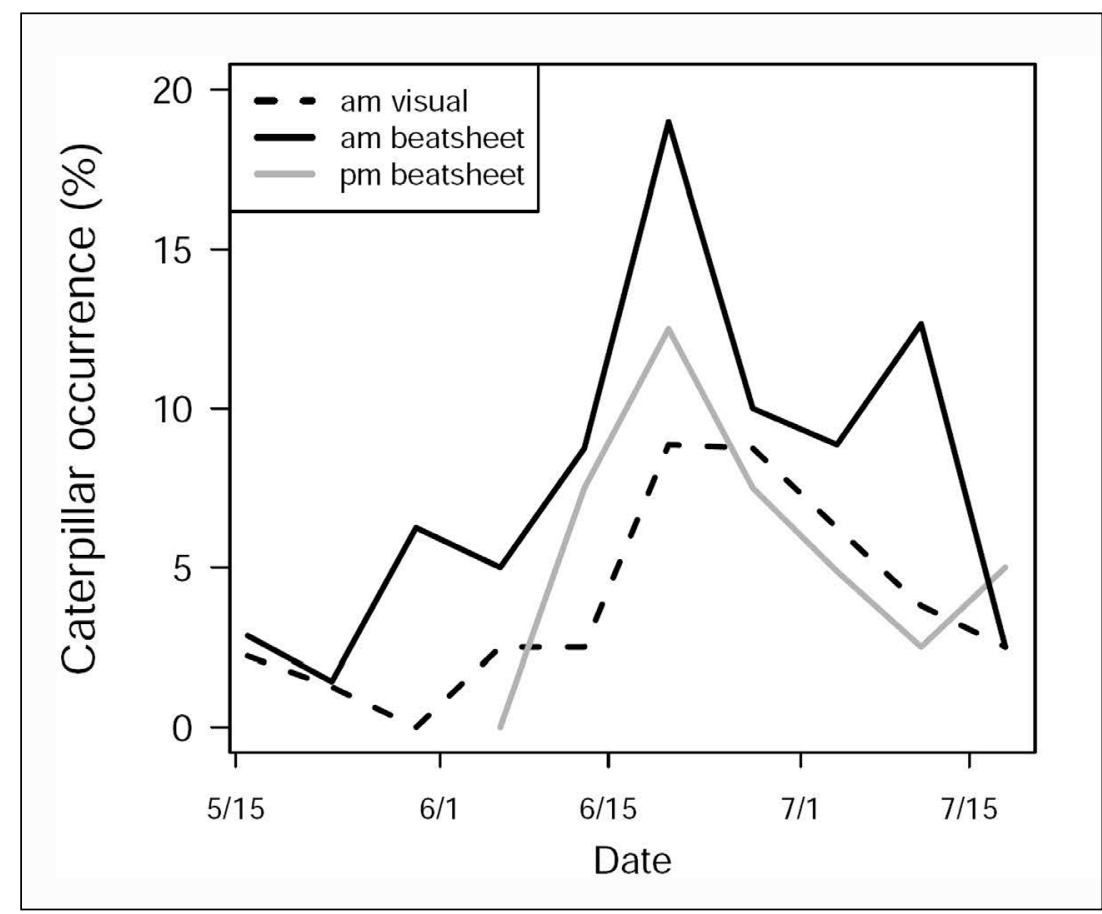

Figure 2: Comparison of caterpillar phenology (fraction of surveys on which at least one caterpillar was detected) at Prairie Ridge Ecostation in 2015 based on morning beatsheet surveys (black solid line), morning visual surveys (black dashed line), and afternoon beatsheet surveys (gray solid line). 
science protocol actually captures the phenology reflected by the frass. Frass traps consisted of a $20 \mathrm{~cm}$ diameter plastic funnel mounted onto a garden stake $30 \mathrm{~cm}$ above ground level and lined with a $40 \mathrm{~cm}$ diameter piece of filter paper folded into a cone. Each frass trap samples a cross-sectional area of $434 \mathrm{~cm}^{2}$. Frass traps were located within existing survey circles ( 1 trap per circle) such that they spanned the same locations as the arthropod surveys. Although frass traps were collected and reset every 3-4 days, data were unusable on dates where there had been major rainstorms since the traps were deployed.

All data were recorded on paper data sheets (although a free mobile app is now available and recommended), and later entered into the project database via a web form. The form limited the entry of extreme values or outliers and also ensured standardization of arthropod names. At the end of each survey day, automated algorithms tallied the number of surveys entered and flagged instances of potentially missing data sheets that had not yet been entered.

\section{Data analysis}

Although all arthropods at least $2 \mathrm{~mm}$ in length were recorded by our protocols, we only used observations of arthropods $5 \mathrm{~mm}$ long or longer in analyses. This reduces the incidence of misidentification of very small individuals, and also minimizes the effect of error in estimating the $2 \mathrm{~mm}$ cutoff. Comparisons of relative arthropod composition between survey methods and between survey participant groups was conducted using chi-squared analyses, while comparisons of absolute density (number observed per survey) across all arthropod groups were conducted using Pearson's correlation coefficients.

Phenology was characterized by the fraction of surveys (occurrence) on which a focal arthropod group was detected on a given date. We used occurrence rather than mean density estimates because the latter are sensitive to outliers, and we had a few instances in which a large number of gregarious caterpillars were observed in a single survey. Because citizen scientists typically collected data only once per week, we averaged the bi-weekly samples of trained scientists into weekly estimates in order to visually compare phenology and calculate Pearson's correlation coefficients across weeks.

To assess the impact of sampling intensity and sampling frequency on estimates of peak caterpillar phenology date, we used data from 2015 where trained scientists conducted 60 beat sheet surveys twice per week from mid-May through mid-July. We fit a Gaussian curve to these data (excluding the last two dates in July which reflect a late season peak less relevant for the avian breeding season; see Figure 5a below) and assumed the estimated mean of this curve reflected the "true" peak date (julian day 172). We then randomly subsampled the full dataset by manipulating both the number of surveys examined per sampling date $(10,20,30,40,50$, or 60 out of the 60 surveys) and the sampling frequency (every sampling date used, every other, every third, every fourth, and every fifth). For each combination of survey number and sampling frequency we conducted 60 replicate subsamples evenly split across potential starting dates (i.e., if sampling frequency was set at every other sampling date, we subsampled using the 1st, 3rd, 5th, etc. dates, but as another replicate also the 2nd, 4th, 6th, etc.). We estimated the peak date from Gaussian fits to the subsampled data. Fits were used only if the mean date was between julian days 100 and 200, and if the $\mathrm{R}^{2}$ for the fit was $>0.2$ ( $89 \%$ of all fits).

\section{Results}

\section{Beat sheet versus visual surveys}

Relative and absolute density estimates for each arthropod group depended upon survey method (Figure 3; $\left.\chi^{2}=284.73, \mathrm{df}=6, p<10^{-16}\right)$. Beat sheet surveys by trained scientists revealed a greater proportion of Coleoptera (beetles) and a lower proportion of Diptera (flies) compared to visual surveys by trained scientists at the same sites and years. A comparison of absolute densities reveals

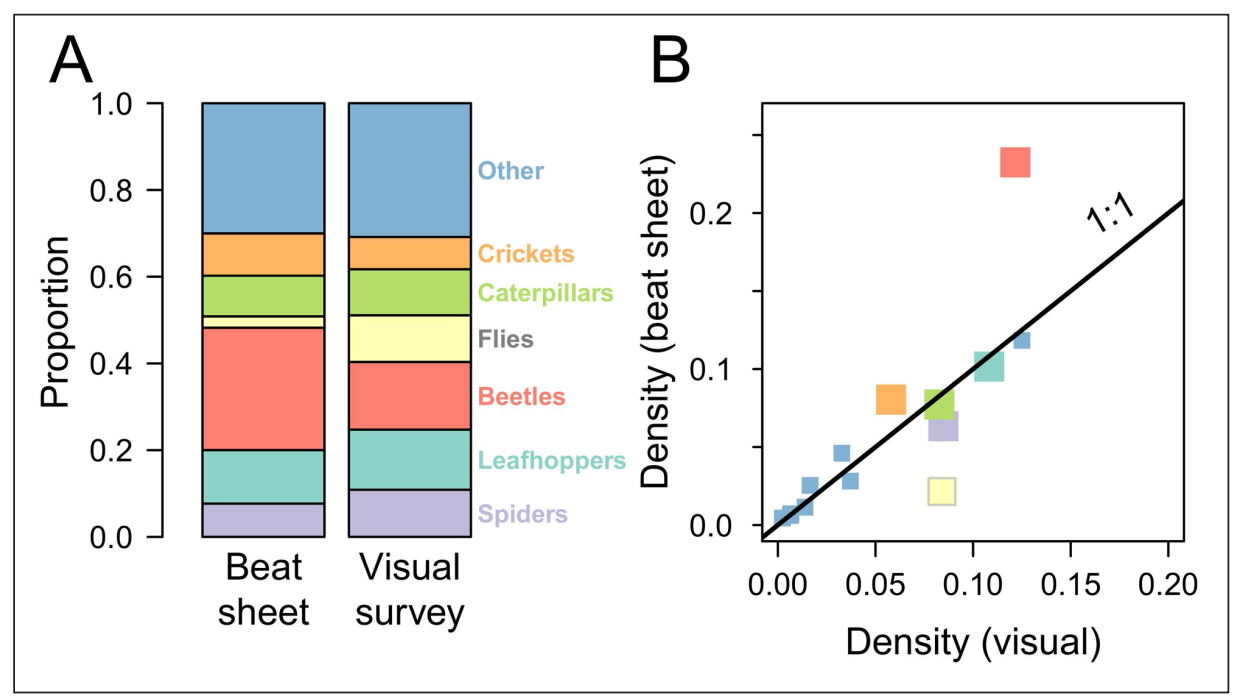

Figure 3: (a) Variation in the proportion of arthropod groups by survey methodology. (b) Comparison of absolute density estimates of different arthropod groups based on survey methodology. Data were collected by trained scientists in both 2015 and 2016. (A comparison of caterpillar phenology observed by these two methods is presented in Figure 2.) 
the same discrepancy with respect to the rates at which beetles and flies are observed using the two methods, but also illustrates that density estimates are comparable for most other arthropod groups $(r=0.82, p=0.0004)$. Notably, caterpillar density estimates were similar using both methods ( 0.077 versus 0.083 caterpillars/survey for beat sheet and visual surveys, respectively).

\section{Citizen scientist versus trained scientist observations: Density}

Perceived arthropod composition differed between citizen scientist- and trained scientist- conducted visual surveys (Figure 4a, $\chi^{2}=44.94$, df $\left.=6, p<5 \mathrm{e} 10^{-8}\right)$. Citizen scientists reported a greater proportion of flies and beetles and a smaller proportion of Auchenorrhyncha (leafhoppers, planthoppers, etc.), Orthoptera (grasshoppers and crickets), and caterpillars compared to the trained scientists, however all differences were within $+/-6 \%$. Absolute density estimates across arthropod taxa were positively correlated between the two groups (Figure 4c triangles, $r=0.84, p<0.0002$ ), and although citizen scientists overestimated fly and beetle density and underestimated caterpillar density relative to trained scientists, these differences were all within 0.05 arthropods/survey.

Using beat sheet surveys, the difference between citizen scientists and trained scientists was less pronounced (Figure $\mathbf{4 b}, \chi^{2}=18.34, \mathrm{df}=6, p=0.005$ ), with citizen scientists reporting a slightly greater proportion of Diptera and Araneae and a slightly lower proportion of Auchenorrhyncha and Coleoptera relative to trained scientists. Again, all differences were within $+/-6 \%$. Absolute density estimates were even more strongly correlated across arthropod taxa between the two groups than in the visual survey comparison (Figure 4c, rectangles, $r=0.94$, $p<0.0001)$. There was much better congruence in estimates of caterpillar and Orthopteran density in particular using beat sheet surveys compared to visual surveys.

\section{Citizen scientist versus trained scientist observations: Phenology}

A primary goal of the Caterpillars Count! project is to characterize the seasonal fluctuations in arthropods over the spring and summer. The phenology of caterpillars as cap- tured by visual and beat sheet surveys near ground level mirrored the phenology of frass falling from the canopy (Figure 5a, dotted line).

As expected, arthropods like caterpillars and orthopterans that depend on leaves for food and shelter exhibited low densities in early spring and then increased over the summer (Figure $\mathbf{5 a - d}$ ). Orthopterans continued to increase through mid- to late-July, while caterpillars exhibited a peak in occurrence in mid-June, followed by another in early July. Foliage arthropods in aggregate (caterpillars, orthopterans, beetles, spiders, leafhoppers, and true bugs) exhibit a general positive trend over the dates examined, with less pronounced seasonal peaks due to the more consistent occurrence of some of those other groups like spiders.

In 2015 using visual surveys, citizen scientists underestimated the occurrence of foliage arthropods early in the season relative to trained scientists, but estimates converged later in the season (Figure 5a, c, e). Citizen scientists did not observe many caterpillars at all until July. As such, they missed the peak in caterpillar occurrence documented by trained scientists in mid-June, although their observations of a decline in mid-July and subsequent recovery in late July were generally consistent (Figure 5a). Similarly, citizen scientists in 2015 also missed the late June peak in orthopterans but captured the peak in July (Figure 5c).

In 2016, using beat sheet surveys, the phenology recorded by citizen scientists was much more strongly correlated with trained scientist observations $(0.50<r<0.95$; Figure $\mathbf{5 b}, \mathbf{d}, \mathbf{f})$. In particular, citizen scientists identified the same increase and mid-June peak in caterpillar occurrence as trained scientists. Citizen scientists did not actually conduct surveys the week of Julian day 186 when trained scientists identified a second seasonal peak in caterpillars.

\section{Sampling frequency and intensity}

Estimates of peak caterpillar date were unbiased with respect to the "true" value (Julian day 172) even at low sampling intensity or frequency (Figure 6). However, as expected, 95\% confidence intervals around the estimated value were tightest when conducting many surveys at

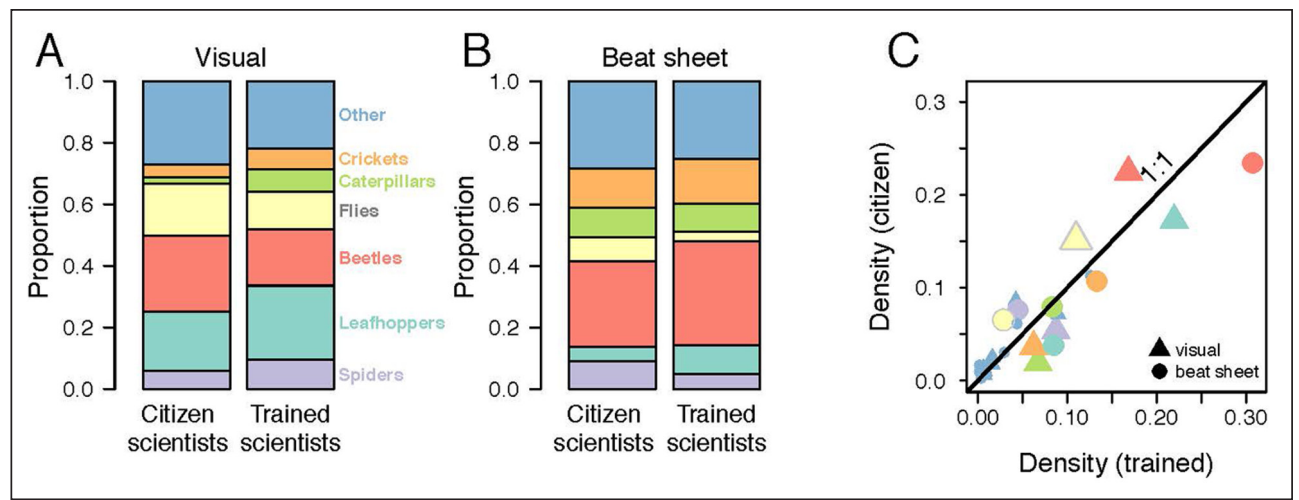

Figure 4: Comparison of the proportion of arthropods observed by citizen scientists versus trained scientists using (a) visual surveys in 2015 and (b) beat sheet surveys in 2016. (c) Comparison of absolute density estimates of different arthropod groups based on whether the data were collected by citizen or trained scientists. 


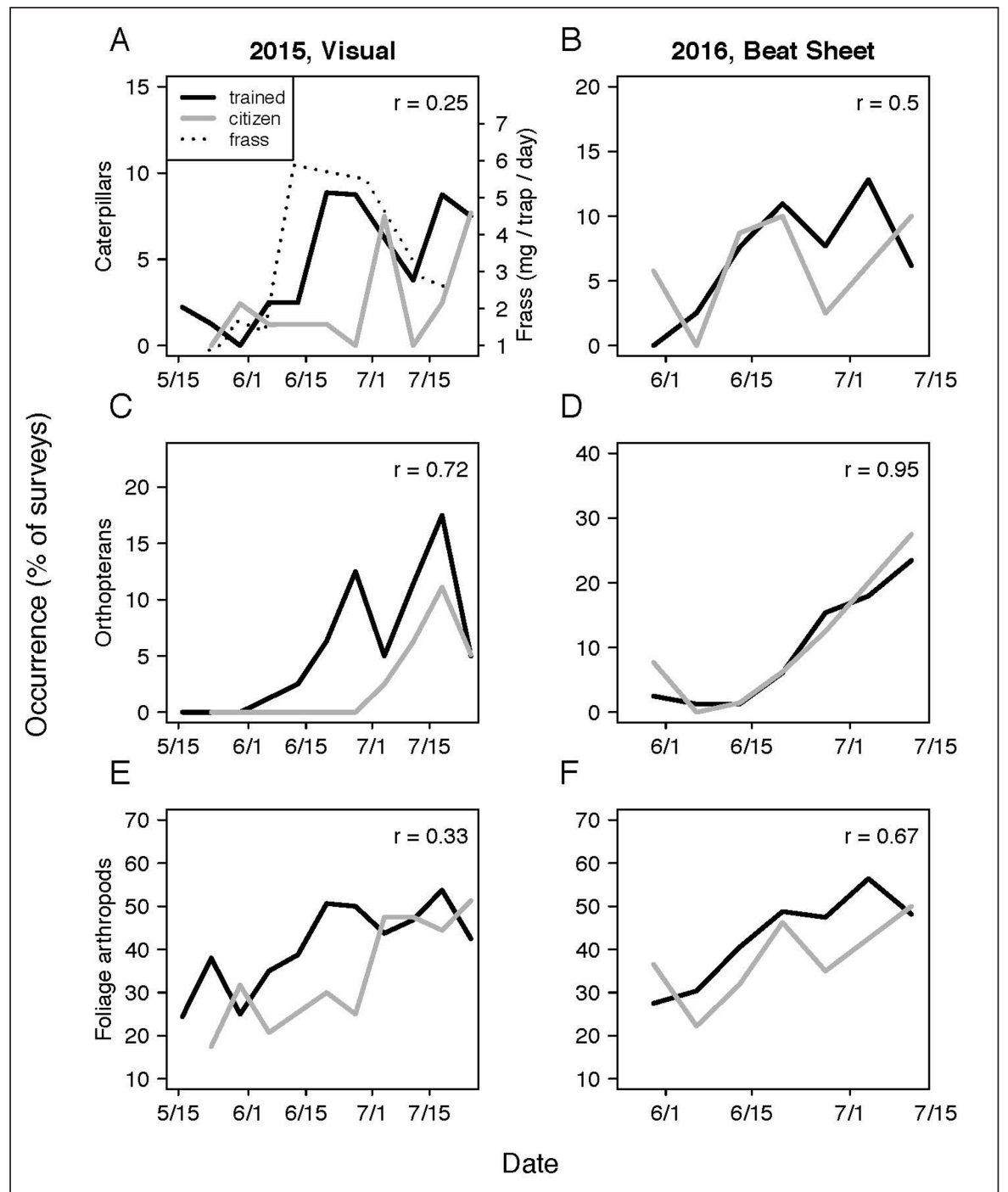

Figure 5: Seasonal phenology in occurrence at Prairie Ridge Ecostation of (a, b) caterpillars, (c, d) orthopterans, and $(\mathbf{e}, \mathbf{f})$ a multi-group category including caterpillars, orthopterans, beetles, spiders, leafhoppers, and true bugs based on visual surveys (a, c, e) and beat sheet surveys (b, d, f). Pearson's correlation coefficient between weekly estimates collected by citizen scientists (light gray) and trained scientists (black) given in the top right. Caterpillar frass phenology in 2015 is shown for comparison in (a).

high frequency, or with a low sampling interval. Estimates of peak caterpillar date based on only a small number of surveys or a low frequency of sampling resulted in estimates that were often weeks from the "true" value. In this particular dataset, sampling 30 surveys on a weekly basis led to $95 \%$ of estimated peak dates falling within one week of the true date. Increasing the number of surveys conducted per sampling date typically yielded a greater increase in accuracy of the peak date estimate compared to increasing the sampling frequency (Figure 6). For example, doubling the number of weekly surveys from 20 to 40 reduced the confidence interval width by more than $50 \%$ (19 days to 9 ), compared to conducting 20 surveys at double the frequency (19 days to 12 ).

\section{Discussion}

Foliage arthropod surveys have the potential to shed light on an important and understudied aspect of ecosystem phenology. However, phenology is expected to vary dra- matically between regions (Both et al. 2004; Hurlbert and Liang 2012) and even across local land use gradients (Diamond et al. 2014; White et al. 2002), necessitating the collection of phenology data across broad geographic scales. Here, we have demonstrated the potential value of enlisting citizen scientists to collect such data, which could greatly facilitate broad-scale investigations into the wide-ranging impacts of climate change on natural systems. In particular, such data would allow researchers to better interpret the consequences of observed phenological shifts by birds that depend on those arthropod resources (Hurlbert 2016; Hurlbert and Liang 2012; Mayor et al. 2017), and shifts by trees and shrubs on which those arthropods depend (Polgar and Primack 2011; Singer and Parmesan 2010). These data would also provide a monitoring baseline for assessing arthropod abundance into the future in light of dramatic population declines reported for many groups from across the globe (Dirzo et al. 2014; Hallmann et al. 2017). These preliminary results help 


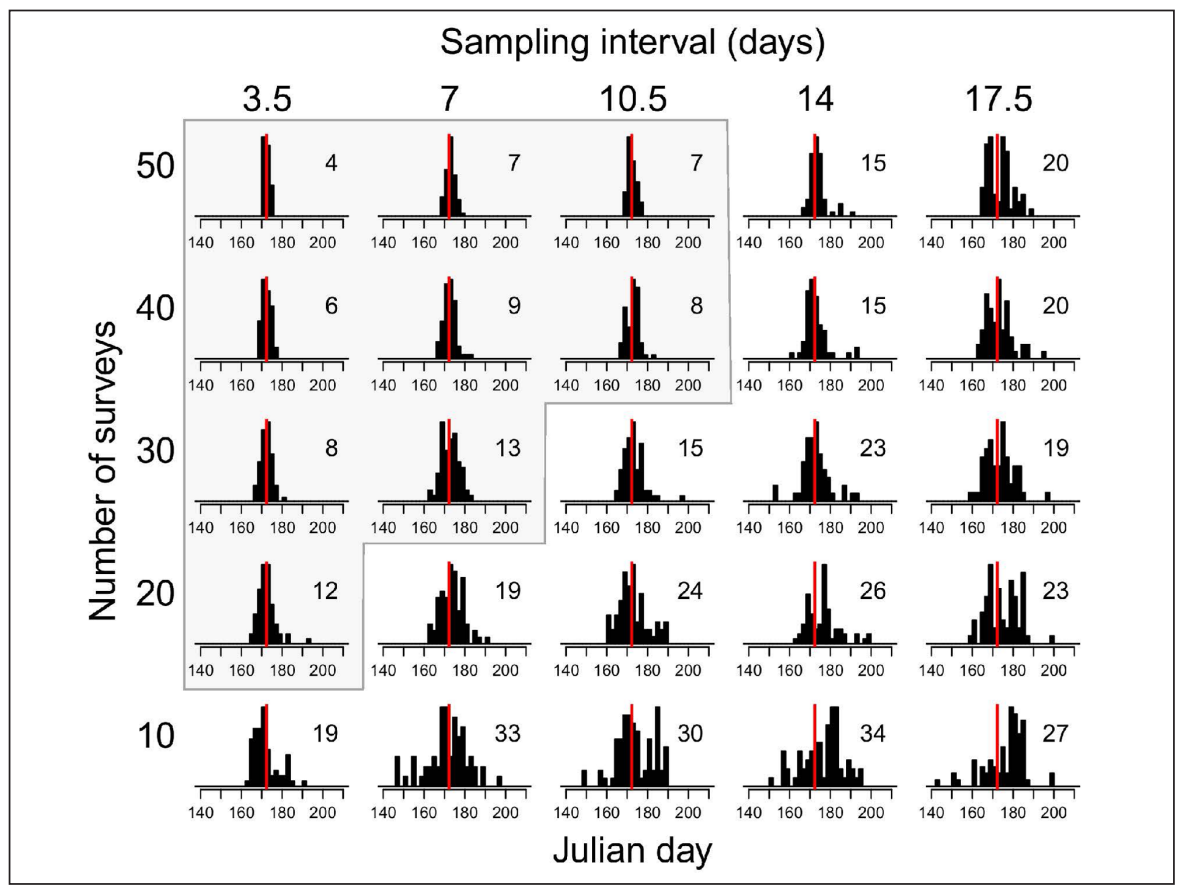

Figure 6: Estimates of peak caterpillar date based on subsampling the trained scientist beat sheet dataset of 2015 to different levels of sampling intensity (rows) and sampling frequency (columns). The "true" estimated peak date based on conducting 60 surveys twice a week was Julian day 172 (June 21; red line). Each histogram indicates the range of peak date estimates based on 60 replicate subsamples for the specified level of sampling frequency and intensity, with the $95 \%$ confidence interval width in days in the upper right corner. Sampling combinations in the gray box have confidence intervals of 13 days or less.

inform the best practices for the Caterpillars Count! survey scheme that will allow researchers to robustly identify patterns of foliage arthropod density in time and space.

\section{Implications for survey methods}

We evaluated two methods for conducting foliage arthropod surveys, visual surveys and beat sheet surveys. In general, the two survey methods yielded very similar results with respect to relative and absolute estimates of arthropod group density based on data collected by trained scientists. As expected, however, each method had its own biases. Flies (Diptera) were underrepresented on beat sheet surveys compared to visual surveys as they tended to fly immediately up and away as soon as a branch was first struck. In contrast, beetles (Coleoptera) were more numerous in beat sheet surveys than in visual surveys. Many of the beetles observed in beat sheets were narrow brownish click beetles (family Elateridae) which rest flat along twigs. This comparison suggests that observers may frequently be overlooking these beetles in visual surveys, although they are quite obvious when lying in a beat sheet. Density estimates for most other groups, including caterpillars, were similar using the two methods. This is interesting given anecdotal observations that some caterpillars, especially those in leaf rolls or sewn between two leaves, are not dislodged by beating, while caterpillars that are extremely cryptic in appearance are more likely to be missed in visual surveys. Although these two groups seemed to be of equivalent abundance such that our two density estimates were comparable, this may not always be the case. Researchers using these data spe- cifically for density estimates will certainly want to take survey method and associated biases into account during analysis. However, phenological metrics of timing which rely on relative, not absolute, indices of abundance should be unbiased.

We found a striking concordance between our ground level survey-based estimates of caterpillar phenology and the canopy level frass-based phenology, suggesting that foliage arthropod surveys conducted near ground level can be used to assess the phenology of higher vegetation strata as well. This correspondence in phenology is consistent with other studies that have found a correlation between lower and upper canopy caterpillar density across trees, years, and season (Cooper 1988; Holmes and Schultz 1988). Monitoring frass phenology at sites where the Caterpillars Count! project is implemented will continue to improve our understanding of where and when phenology varies across forest strata, and we are currently exploring how frass monitoring may form the basis for a complementary citizen science project.

\section{Agreement between citizen scientists and trained scientists}

For both survey methods, citizen scientists generated relative density estimates that were, on average, within $6 \%$ of those generated by trained scientists. Beat sheet surveys yielded stronger agreement between citizen scientists and trained scientists with respect to density estimates and phenology compared to visual surveys. This was especially true for caterpillars: In 2015 citizen scientists entirely missed the mid-June peak in caterpillar occurrence when 
conducting visual surveys, while the citizen scientists in 2016 documented patterns similar to the trained scientists using beat sheet surveys. The individual citizen scientist participants differed between 2015 and 2016 and numbered only 10 unique individuals in total, indicating that this effect is just as likely to be a participant effect as a survey method effect. Anecdotally, one participant in 2015 was notably less engaged and motivated compared to participants in 2016, highlighting the need to further validate the use of visual surveys in this project. Certainly, not all participants would necessarily have missed the caterpillar peak in 2015. Nevertheless, the ideal arthropod survey methodology is one that is robust to variation in participant ability and motivation. The task of detecting arthropods against a white beat sheet is presumably less subject to error than that of detecting arthropods on an often similarly colored branch. Given a choice, we therefore encourage citizen science participants to use beat sheets if possible.

Another advantage of beat sheet surveys in the context of citizen science is the ability to engage and involve younger participants. Although children are not the target participant group for this project, beat sheet surveys require considerably less time and patience than visual surveys, and may be better for youth education programs. Beat sheets are also useful for displaying interesting arthropods to a group, providing an unobstructed view and avoiding the need to have observers step up to a branch one at a time. Although constructing a homemade beat sheet is fairly simple and cheap (approximately $\$ 5$ in fabric and hardware), it still represents a potential barrier for participants or environmental education centers with limited resources. For that reason alone, we expect that some local project coordinators will choose to conduct visual surveys. Our comparison of the two methods provides an initial suggestion of how to compare data obtained in each, but conducting this methods comparison in other habitats and regions would be useful.

\section{Implications for sampling frequency and intensity}

Finally, we examined how variation in sampling intensity and frequency influenced the perceived date of peak caterpillar occurrence. This is an important question because citizen scientist participants have finite time and resources to dedicate to any particular project. While estimates of phenology become more precise with increased data collection, the number of participants willing to meet those increased data collection requirements will be smaller (Sauermann and Franzoni 2015). We found that conducting 30 foliage surveys on a weekly basis provided estimates of peak caterpillar occurrence typically within 1 week of the "true" peak, and we recommend this level of effort as a best practice. If a greater sampling effort is possible, increasing the number of surveys conducted per sampling date yields a greater increase in precision of phenological estimates compared to investing an equivalent amount of effort in increased sampling frequency and so should be preferred. Put another way, conducting 40 surveys once a week is better than visiting the same 20 surveys twice each week. A smaller number of surveys may still be useful in assessing phenology in a qualitative sense (e.g., determining whether it's an "early" or "late" year). We will more rigorously evaluate this possibility as we accumulate more years of survey data.

Because a single foliage survey by an untrained individual conservatively takes about 6 minutes (including sharing observations with others, walking between surveys, etc.), our recommended effort (30 surveys) requires 3 person-hours per week. While some dedicated and interested individuals may participate at this level, they will be in the minority. For this reason, Caterpillars Count! will be most easily carried out at centralized locations such as environmental education centers that frequently host thousands of visitors each season and have groups of dedicated, regular volunteers eager to contribute toward projects at the site. At centers like these, the data collection effort can be divided among several people such that, for example, a group of 5 could conduct 30 surveys in less than forty minutes. In this way, individuals interested in participating for only a single day may still contribute to the project within a discrete amount of time and with the assistance of trained and experienced participants. This distributed effort strategy still requires one individual at the site who can coordinate the efforts of other participants, and our experience at Prairie Ridge Ecostation suggests that this will require 2 hours per week once the project is up and running.

\section{Sources of error and bias}

Data collection for this project involves three potential sources of error in the context of phenology estimation. First, participants must detect arthropods on survey branches or beat sheets. As discussed above, detectability is expected to be a greater problem for visual surveys due to crypsis, although detectability on beat sheets may still be an issue for arthropods that fly, jump, or run out of the sheet before they can be observed. Nevertheless, for detectability to bias phenological signal, it must vary systematically over time. This may be less of an issue for beat sheet surveys, however, the ability to detect insects on branches via visual surveys almost certainly increases with experience. For sites at which the same individual or individuals conduct visual surveys each week, one might expect observations in the first few survey periods to underestimate arthropod occurrence relative to later in the season. Quantifying exactly how arthropod searching ability improves over time will help to determine whether this bias is mostly eliminated after a single day of conducting 5-10 surveys, or if it is likely to persist over a longer period. Nevertheless, to the extent that seasonal arthropods decline in late summer (e.g., July for caterpillars at our study sites), this phenomenon should be well captured by observers regardless of any increases in searching competence.

Second, participants must properly identify arthropods to the appropriate group (Table 1). For groups like caterpillars and spiders, this task will be straightforward. Distinguishing beetles from true bugs and leafhoppers may be more prone to error. We have developed outreach materials including identification keys and cheatsheets to 
assist participants while they are in the field). We have also developed an arthropod photo identification quiz, which is on our website (https://caterpillarscount.unc.edu/arthropodQuiz/). The quiz may be taken repeatedly with different photos of common foliage arthropods each time. Finally, although not used for this study, Caterpillars Count! now features a free mobile app for data submission. With the app, users may optionally photograph the arthropods they encounter, and these photographs will be automatically submitted to the crowdsourcing identification website iNaturalist.org. This feature allows those who are interested to pursue lower taxonomic level identification by experts.

Third, participants must estimate the body length of arthropods to the nearest millimeter. Although much of the US public is less familiar with metric units, having participants calibrate familiar objects like the width of a fingernail or a pencil is fairly straightforward, and simple rulers can be drawn on the supports of a beat sheet or included in the mobile app and arthropod identification guides. Regardless, errors in length estimation will not impact phenology patterns based on occurrence or density. Even in the event that arthropod lengths are used to calculate biomass phenology via length-weight regressions, length estimates are not expected to be biased seasonally in one direction or the other.

\section{Incentives for participation}

Robust survey protocols are necessary but insufficient for ensuring a citizen science project's success. Equally important are considerations about the motivations and incentives for participating (Hobbs and White 2012), both from the perspective of potential one-time contributors like weekend visitors to an environmental education center, as well as new potential site coordinators and their regular volunteers. Caterpillars Count! provides a context for interested individuals to learn about the natural world around them and to contribute to a broader scientific understanding of arthropod phenology and its consequences in a changing world. The project also provides participants who have affinities to particular Caterpillars Count! sites the ability to contribute to something meaningful at that site which advances broader citizen science outcomes (Jordan et al. 2011; McKinley et al. 2017). We hope the availability of arthropod identification resources, mobile apps for easy data collection, data visualization tools on the project website, and structured learning activities associated with the project will provide additional incentives for environmental educators and others to initiate a Caterpillars Count! monitoring scheme that integrates citizen science volunteers. It is our hope that Caterpillars Count! will yield robust data on arthropod phenology over broad spatial scales that can ultimately be leveraged with other existing datasets to provide new insights into potential mismatches between vegetation, arthropods, and birds.

\section{Acknowledgements}

We would like to thank everyone who helped to collect data for this project, including L. Diez, R. Garland, T. Hennessey, W. Larsen, S. Taylor, R. Ward, and T. Wilkins.
We also acknowledge the environmental educators and others who have provided feedback on protocols, mobile apps, and activities. We also acknowledge the constructive feedback provided by $\mathrm{M}$. Katti and four anonymous reviewers. AHH was supported by a grant from NSF Macrosystems Biology EF-1702708.

\section{Competing Interests}

The authors have no competing interests to declare.

\section{References}

Altermatt, F. 2012. Temperature-related shifts in butterfly phenology depend on the habitat. Glob. Change Biol, 18: 2429-2438. DOI: https://doi.org/10.1111/j.13652486.2012.02727.x

Both, C, Artemyev, AV, Blaauw, B, Cowie, RJ, Dekhuijzen, AJ, Eeva, T, Enemar, A, Gustafsson, L, Ivankina, EV, Järvinen, A, Metcalfe, NB, Nyholm, NEI, Potti, J, Ravussin, P-A, Sanz, JJ, Silverin, B, Slater, FM, Sokolov, LV, Török, J, Winkel, W, Wright, J, Zang, H and Visser, ME. 2004. Large-scale geographical variation confirms that climate change causes birds to lay earlier. Proc. R. Soc. Lond. Ser. B-Biol. Sci, 271: 1657-1662. DOI: https://doi.org/10.1098/ rspb.2004.2770

Both, C, Van Asch, M, Bijlsma, RG, Van Den Burg, AB and Visser, ME. 2009. Climate change and unequal phenological changes across four trophic levels: Constraints or adaptations? J. Anim. Ecol, 78: 73-83. DOI: https://doi.org/10.1111/j.1365-2656.2008.01458.x

Cooper, RJ. 1988. Dietary relationships among insectivorous birds of an eastern deciduous forest (Ph.D.). West Virginia University, Morgantown, WV.

Diamond, SE, Cayton, H, Wepprich, T, Jenkins, CN, Dunn, RR, Haddad, NM and Ries, L. 2014. Unexpected phenological responses of butterflies to the interaction of urbanization and geographic temperature. Ecology. DOI: https://doi.org/10.1890/13-1848.1

Dirzo, R, Young, HS, Galetti, M, Ceballos, G, Isaac, NJB and Collen, B. 2014. Defaunation in the Anthropocene. Science, 345: 401-406. DOI: https://doi. org/10.1126/science. 1251817

Ellwood, ER, Temple, SA, Primack, RB, Bradley, NL and Davis, CC. 2013. Record-Breaking Early Flowering in the Eastern United States. PLOS ONE, 8: e53788. DOI: https://doi.org/10.1371/journal.pone.0053788

Fontaine, J, Stutzman, R and Gannes, L. 2015. Leaps, Chains, and Climate Change for Western Migratory Songbirds. In: Phenological Synchrony and Bird Migration, Studies in Avian Biology. CRC Press, 3-16. DOI: https://doi.org/10.1201/b18011-3

Forister, ML and Shapiro, AM. 2003. Climatic trends and advancing spring flight of butterflies in lowland California. Glob. Change Biol, 9: 1130-1135. DOI: https://doi.org/10.1046/j.1365-2486.2003.00643.x

Forrest, JRK. 2015. Plant-pollinator interactions and phenological change: What can we learn about climate impacts from experiments and observations? Oikos, 124: 4-13. DOI: https://doi.org/10.1111/oik.01386 
Graber, JW and Graber, RR. 1983. Feeding rates of warblers in spring. The Condor, 85: 139-150. DOI: https:// doi.org/10.2307/1367247

Hallmann, CA, Sorg, M, Jongejans, E, Siepel, H, Hofland, N, Schwan, H, Stenmans, W, Müller, A, Sumser, H, Hörren, T, Goulson, D and de Kroon, H. 2017. More than 75 percent decline over 27 years in total flying insect biomass in protected areas. PLOS ONE, 12: e0185809. DOI: https://doi.org/10.1371/ journal.pone.0185809

Hobbs, SJ and White, PCL. 2012. Motivations and barriers in relation to community participation in biodiversity recording. J. Nat. Conserv, 20: 364-373. DOI: https://doi.org/10.1016/j.jnc.2012.08.002

Holmes, RT, Bonney, RE and Pacala, SW. 1979. Guild structure of the Hubbard Brook bird community: A multivariate approach. Ecology, 60: 512-520. DOI: https://doi.org/10.2307/1936071

Holmes, RT and Schultz, JC. 1988. Food availability for forest birds: Effects of prey distribution and abundance on bird foraging. Can. J. Zool, 66: 720-728. DOI: https://doi.org/10.1139/z88-107

Hurlbert, AH. 2016. Birds are shifting migration timing, but how, why, and is it enough? Ecology, 97: 2171-2172. DOI: https://doi.org/10.1002/ecy.1509

Hurlbert, AH and Liang, Z. 2012. Spatiotemporal Variation in Avian Migration Phenology: Citizen Science Reveals Effects of Climate Change. PLOS ONE, 7: e31662. DOI: https://doi.org/10.1371/journal. pone.0031662

Johnson, KA. 2016. Real Life Science with Dandelions and Project BudBurst. J. Microbiol. Biol. Educ, 17: 115-116. DOI: https://doi.org/10.1128/jmbe.v17i1.1064

Jones, J, Doran, PJ and Holmes, RT. 2003. Climate and Food Synchronize Regional Forest Bird Abundances. Ecology, 84: 3024-3032. DOI: https://doi. org/10.2307/3449971

Jordan, RC, Gray, SA, Howe, DV, Brooks, WR and Ehrenfeld, JG. 2011. Knowledge gain and behavioral change in citizen-science programs. Conserv. Biol. J. Soc. Conserv. Biol, 25: 1148-1154. DOI: https://doi. org/10.1111/j.1523-1739.2011.01745.x

Mayor, SJ, Guralnick, RP, Tingley, MW, Otegui, J, Withey, JC, Elmendorf, SC, Andrew, ME, Leyk, S, Pearse, IS and Schneider, DC. 2017. Increasing phenological asynchrony between spring green-up and arrival of migratory birds. Sci. Rep, 7: 1902. DOI: https://doi.org/10.1038/s41598-017-02045-z

McKinley, DC, Miller-Rushing, AJ, Ballard, HL, Bonney, R, Brown, H, Cook-Patton, SC, Evans, DM, French, RA, Parrish, JK, Phillips, TB, Ryan, SF, Shanley, LA, Shirk, JL, Stepenuck, KF, Weltzin, JF, Wiggins, A, Boyle, OD, Briggs, RD, Chapin, SF, Hewitt, DA, Preuss, PW and Soukup, MA. 2017. Citizen science can improve conservation science, natural resource management, and environmental protection. Biol. Conserv., The role of citizen science in biological conservation, 208: 15-28. DOI: https://doi.org/10.1016/j. biocon.2016.05.015
Nagy, LR and Holmes, RT. 2005a. Food limits annual fecundity of a migratory songbird: An experimental study. Ecology, 86: 675-681. DOI: https://doi. org/10.1890/04-0155

Nagy, LR and Holmes, RT. 2005b. To Double-Brood or Not? Individual Variation in the Reproductive Effort in Black-Throated Blue Warblers (Dendroica caerulescens) (¿Poner Nidadas Dobles o No? Variación Individual en el Esfuerzo Reproductivo en Dendroica caerulescens). The Auk, 122:902-914. DOI:https://doi.org/10.1642/00048038(2005)122[0902:TDONIV]2.0.CO;2

Parmesan, C. 2007. Influences of species, latitudes and methodologies on estimates of phenological response to global warming. Glob. Change Biol, 13: 1860-1872. DOI: https://doi.org/10.1111/j.13652486.2007.01404.x

Parmesan, C and Yohe, G. 2003. A globally coherent fingerprint of climate change impacts across natural systems. Nature, 421: 37-42. DOI: https://doi. org/10.1038/nature01286

Perrins, CM. 1991. Tits and their caterpillar food supply. Ibis, 133: 49-54. DOI: https://doi.org/10.1111/j.1474919X.1991.tb07668.x

Polgar, CA and Primack, RB. 2011. Leaf-out phenology of temperate woody plants: From trees to ecosystems. New Phytol, 191: 926-941. DOI: https://doi. org/10.1111/j.1469-8137.2011.03803.x

Primack, RB. 2014. Walden Warming: Climate change comes to Thoreau's woods. University of Chicago Press, Chicago. DOI: https://doi.org/10.7208/chicago/9780226062211.001.0001

Rodenhouse, NL and Holmes, RT. 1992. Results of experimental and natural food reductions for breeding Black-throated Blue Warblers. Ecology, 73: 357-372. DOI: https://doi.org/10.2307/1938747

Sauermann, H and Franzoni, C. 2015. Crowd science user contribution patterns and their implications. Proc. Natl. Acad. Sci, 112: 679-684. DOI: https://doi. org/10.1073/pnas.1408907112

Schwartz, MD, Betancourt, JL and Weltzin, JF. 2012. From Caprio's lilacs to the USA National Phenology Network. Front. Ecol. Environ, 10: 324-327. DOI: https://doi.org/10.1890/110281

Sillett, TS, Holmes, RT and Sherry, TW. 2000. Impacts of a global climate cycle on population dynamics of a migratory songbird. Science, 288: 2040-2042. DOI: https://doi.org/10.1126/science.288.5473.2040

Singer, MC and Parmesan, C. 2010. Phenological asynchrony between herbivorous insects and their hosts: Signal of climate change or pre-existing adaptive strategy? Philos. Trans. R. Soc. B Biol. Sci, 365: 3161-3176. DOI: https://doi.org/10.1098/rstb.2010.0144

Sullivan, BL, Aycrigg, JL, Barry, JH, Bonney, RE, Bruns, N, Cooper, CB, Damoulas, T, Dhondt, AA, Dietterich, T, Farnsworth, A, Fink, D, Fitzpatrick, JW, Fredericks, T, Gerbracht, J, Gomes, C, Hochachka, WM, Iliff, MJ, Lagoze, C, La Sorte, FA, Merrifield, M, Morris, W, Phillips, TB, Reynolds, M, Rodewald, AD, Rosenberg, KV, Trautmann, NM, 
Wiggins, A, Winkler, DW, Wong, W-K, Wood, CL, Yu, J and Kelling, S. 2014. The eBird enterprise: An integrated approach to development and application of citizen science. Biol. Conserv, 169: 31-40. DOI: https://doi.org/10.1016/j.biocon.2013.11.003

Visser, M, Holleman, L and Gienapp, P. 2006. Shifts in caterpillar biomass phenology due to climate change and its impact on the breeding biology of an insectivorous bird. Oecologia, 147: 164-172. DOI: https://doi. org/10.1007/s00442-005-0299-6

Visser, ME, te Marvelde, L and Lof, ME. 2012. Adaptive phenological mismatches of birds and their food in a warming world. J. Ornithol, 153: S75-S84. DOI: https://doi.org/10.1007/s10336-011-0770-6

White, MA, Nemani, RR, Thornton, PE and Running, SW. 2002. Satellite evidence of phenological differences between urbanized and rural areas of the eastern United States deciduous broadleaf forest. Ecosystems, 5: 260-273. DOI: https://doi.org/10.1007/ s10021-001-0070-8

Wood, EM and Kellermann, JL. 2015. Phenological synchrony and bird migration: Changing climate and seasonal resources in North America. CRC Press, Boca Raton. DOI: https://doi.org/10.1201/b18011

How to cite this article: Hurlbert, A, Hayes, T, McKinnon, T and Goforth, C. 2019. Caterpillars Count! A Citizen Science Project for Monitoring Foliage Arthropod Abundance and Phenology. Citizen Science: Theory and Practice, 4(1): 1, pp. 1-12. DOI: https:// doi.org/10.5334/cstp.148

Submitted: 26 January 2018 Accepted: 29 October 2018 Published: 23 January 2019

Copyright: ( 2019 The Author(s). This is an open-access article distributed under the terms of the Creative Commons Attribution 4.0 International License (CC-BY 4.0), which permits unrestricted use, distribution, and reproduction in any medium, provided the original author and source are credited. See https://creativecommons.org/licenses/by/4.0/.

] $\mathrm{u}[\quad$ Citizen Science: Theory and Practice is a peer-reviewed open access journal published by Ubiquity Press. 